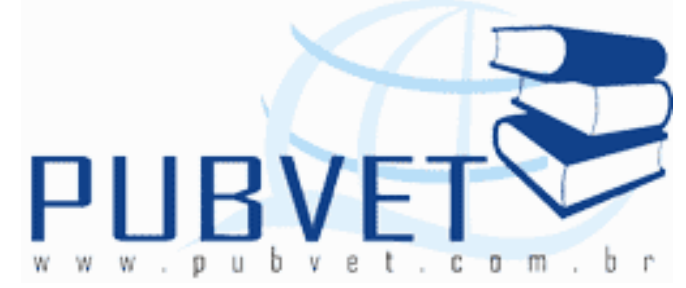

PUBVET, Publicações em Medicina Veterinária e Zootecnia.

\title{
Utilização de métodos didáticos alternativos para o estudo da anatomia veterinária
}

Aline Luize de Moraes Souza1, Rômulo Ferreira de Assumção ${ }^{1}$, Luísa Faustino ${\text { Guimarães }{ }^{1} \text {, Ana Bárbara Freitas Rodrigues² }}^{2}$

${ }^{1}$ Graduando do curso de Medicina Veterinária da Universidade Estadual Norte Fluminense Darcy Ribeiro - UENF

2 Professora de Anatomia Animal do Laboratório de Morfologia e Patologia Animal do Centro de Ciências e Tecnologias Agropecuárias da UENF

\section{Resumo}

Os animais são, rotineiramente, utilizados tanto para fins educacionais quanto para a pesquisa, seu uso ocorre no sentido de repassar informações já adquiridas, principalmente, para o estudo da anatomia animal. Este trabalho tem o propósito de desenvolver modelos didáticos alternativos que possam ser utilizados no ensino da anatomia veterinária. Desta forma, se reduziria o uso rotineiro, em aulas práticas de anatomia veterinária, de peças anatômicas oriundas de animais mortos. Com esse intuito, foram confeccionados os modelos das articulações do ombro e do joelho de cão, utilizando massa de biscuit para representar os diferentes ligamentos existentes nas referidas articulações. A reprodução fiel dos estômagos de eqüino e cão também foi ensaiada utilizando-se os próprios estômagos extraídos de animais que vieram a óbito e, neste experimento, foram preparados com a finalidade de servirem 
como moldes. Todos os estômagos foram previamente tratados com extrato de própolis e em seguida foram preenchidos com serragem, suturados e envernizados. A secagem por injeção de ar, um método de conservação de vísceras ocas, também vem sendo empregado em nosso setor na preparação de estômagos de diferentes espécies animais. A utilização desta técnica nos permite comparar a morfologia do trato digestório de diferentes espécies. Uma análise da topografia vascular da face de um equino pode ser demonstrada com o uso de lã (vermelha) colada sobre a superfície óssea da cabeça do animal, ilustrando desta forma toda trama arterial que é responsável pela irrigação sanguínea desta região. A utilização destes recursos didáticos vem se mostrando um agente facilitador da aprendizagem, contribuindo de maneira positiva no processo ensino-aprendizagem. Assim, é possível concluir que a utilização de métodos alternativos ao uso desnecessário de animais é ético, eficaz e economicamente viável.

Palavras-chave: Modelo didático, anatomia, animal

\title{
Educational use of alternative methods for the study of veterinary anatomy
}

\begin{abstract}
Animals are routinely used both for educational and research as to its use occurs in order to pass on information already acquired mainly for anatomical studies. This work aims to develop alternative teaching models that can be used in the teaching of veterinary anatomy. Thus, the routine use would be reduced in practical classes in veterinary anatomy, anatomical parts derived from dead animals. To that end, the models were made of the shoulder joints and elbow dog biscuit dough using to represent the various structures existing in these joints. The faithful reproduction of the stomachs of horse and dog also was tested using their own stomachs taken from animals that died and came in this experiment were prepared and used as templates. All stomachs were pretreated with propolis extract and then were filled with sawdust, stitched and
\end{abstract}


varnished. The drying air injection, a method of preservation of hollow viscera, also has been used in our industry in the preparation of the stomachs of different animal species. This technique allows us to compare the morphology of the digestive tract of different species. An analysis of the topography of the vascular face of a horse can be demonstrated with the use of wool (red) mounted on the bone surface of the animal's head, thus illustrating the whole plot which is responsible for arterial blood supply to this region. The use of teaching resources has proven to be a facilitator of learning, contributing positively in the teaching-learning. Thus, we conclude that the use of alternative methods to the unnecessary use of animals is ethical, effective and economically viable.

Keywords: teaching model, anatomy, animal

\section{INTRODUÇÃO}

Os animais são, rotineiramente, utilizados tanto para fins educacionais quanto para a pesquisa, seu uso ocorre no sentido de repassar informações já adquiridas, principalmente, para estudos anatômicos (MAGALHÃES, 2006). O uso de animais como recurso didático no ensino superior tem sido muito discutido. Tal debate acerca desse assunto tem aumentado muito nas últimas décadas, enquanto grupos de proteção aos animais tentam convencer muitas universidades a restringirem, e até mesmo abolirem, a utilização de animais com fins didáticos (JUKES, 2004; MAGALHÃES, 2006). Com base nisso, o conceito dos três Rs (reduction, refinement and replacement), enunciados por William Russel e Rex Burch, em 1959, tornou-se um foco tanto para o bem estar animal quanto para as comunidades científicas em todo o mundo. Adesão aos três Rs é agora uma exigência da maioria, se não todos legislados e autoregulados sistemas nacionais de vigilância(GAUTHIER,2005), sendo base para a redução do número de animais utilizados, o aprimoramento das técnicas que os utilizam e a substituição destes. Com relação às técnicas que visam a substituição dos animais, estão inclusos os métodos alternativos de ensino, 
que vêm sendo utilizados no mundo todo. A utilização de técnicas anatômicas e de materiais alternativos pode ser uma forma opcional para produzir peças ou modelos anatômicos, que normalmente são de difícil obtenção através de dissecação, por serem muito pequenas ou de difícil acesso (SANTOS,2010). Nestes casos, tais técnicas tornam-se ferramentas eficientes para produção de modelos anatômicos, com custo relativamente baixo e de fácil aquisição para serem utilizados no processo ensino-aprendizagem do estudo da anatomia animal (SANTOS,2010).

\section{MATERIAL E MÉTODOS}

Foram utilizados estômagos de diferentes espécies, articulações do úmero e do joelho de um cão e um sincrânio de um equino. Todas as peças utilizadas foram de animais que vieram a óbito e em sequência foram doados à seção de anatomia da Universidade Estadual do Norte Fluminense Darcy Ribeiro. Para realização deste trabalho foram utilizadas quatro técnicas distintas com o objetivo de criar peças anatômicas alternativas para o ensino da anatomia animal.

\section{Estômagos preenchidos com forragem}

Os estômagos de cão e equino foram limpos, preparados e preenchidos com serragem. Todas as peças foram modeladas manualmente para que fossem evidenciados detalhes da morfologia externa como a curvatura maior e menor do estômago. Em seguida, cada estômago foi mergulhado em uma solução alcoólica de extrato de própolis, que serviu como agente antimicrobiano, sendo finalmente envernizados para permitir uma melhor conservação das peças (Figura 1). 


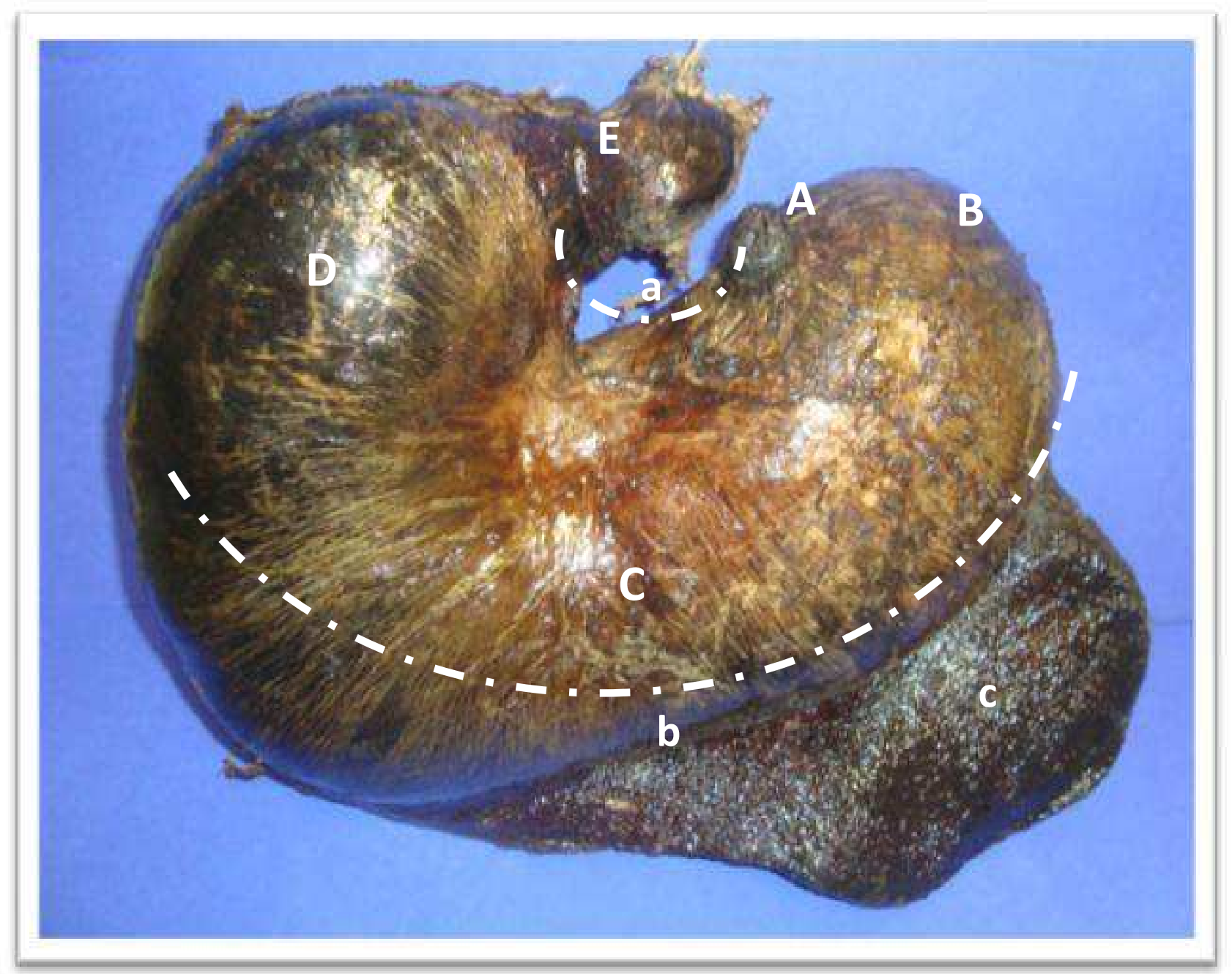

Figura 1: Estômago e baço de equino. A- esôfago, B- fundo do estômago, C- corpo do estômago, D- porção pilórica do estômago, E- canal pilórico, a- curvatura menor, b- curvatura maior e c- baço.

\section{Secagem por injeção de ar}

Consiste em um método de conservação de vísceras ocas. Os estômagos foram previamente dissecados, para evidenciar os detalhes anatômicos, em seguida tiveram os seus lúmens preenchidos com uma solução aquosa de formol $10 \%$ e em seguida foram conservado em geladeira por um dia. Após esse período os estômagos foram acoplados à maquina de secagem por pressão, que 
proporcionou a passagem de ar constante no interior dos estômagos, até que estivessem completamente secos, sendo finalizados com uma camada de verniz para conservação. (Figura 2).
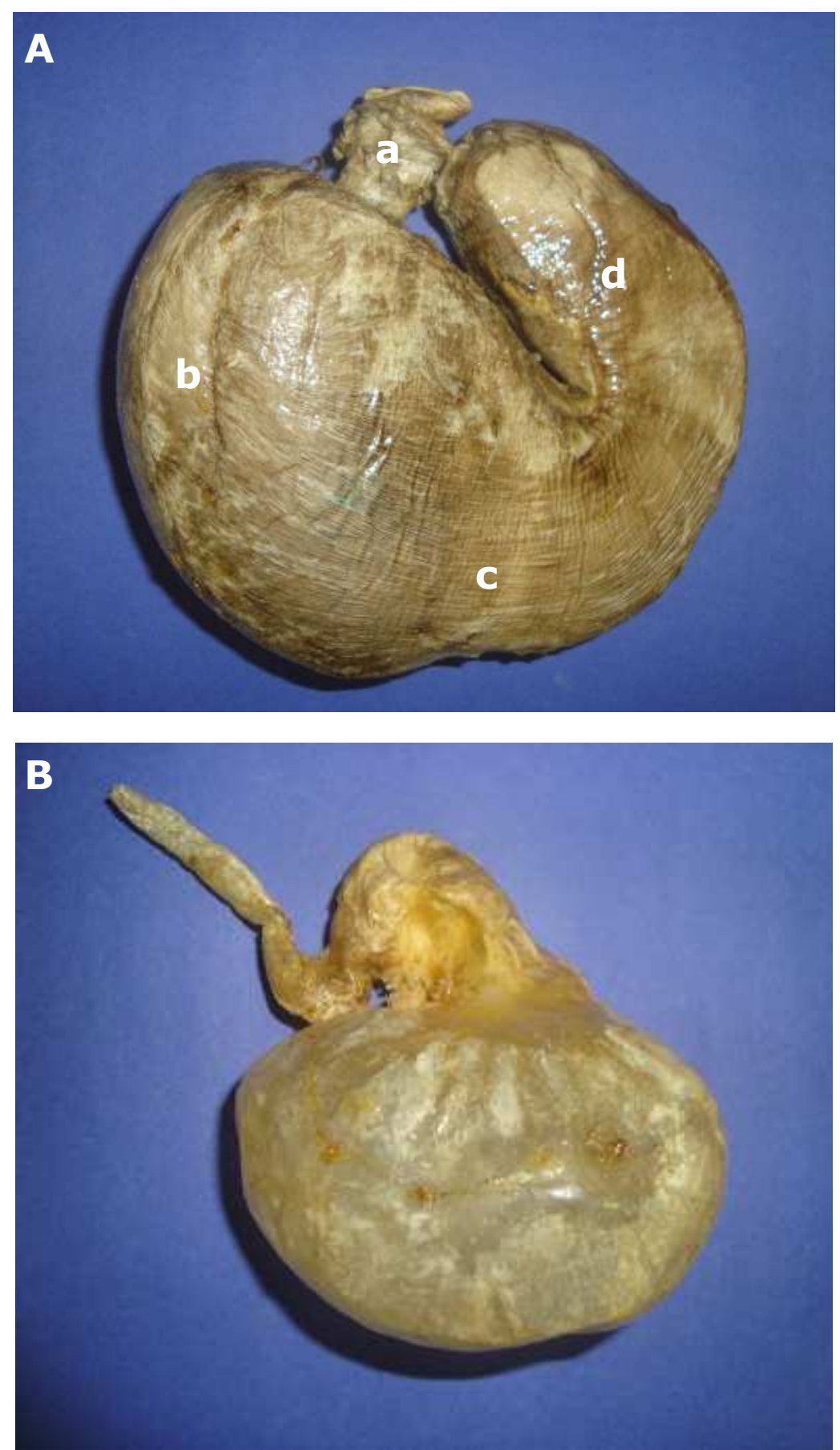

Figura 2: A- Vista visceral do estômago de cão secado a ar: a- Esôfago; b-Fundo do estômago, c- Corpo do estômago, d - Região pilórica. B- Estômago de coelho secado a ar. 


\section{Modelos de articulação}

Foram preparados modelos de articulações utilizando as bases ósseas das articulações dos membros torácico e pélvico de cão. Os ossos que formam as articulações do ombro e do joelho foram unidos e fixados por meio de cola e arame, e os ligamentos correspondestes a estas articulações reproduzidos com o auxílio de massa de biscuit (Figuras 3 e 4).

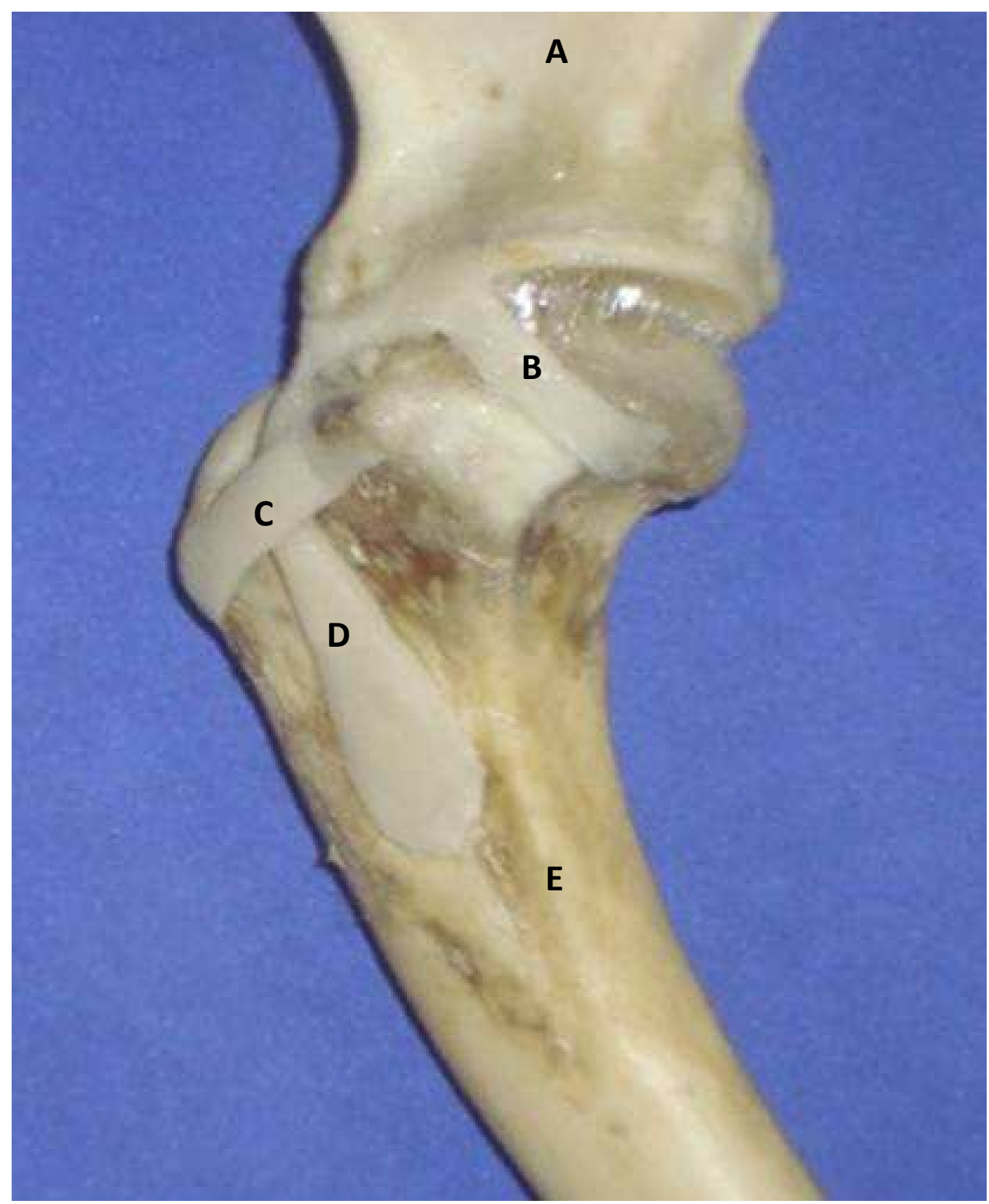

Figura 3- Articulação do ombro de cão: A - escápula; Bligamento glenoumeral medial; C- ligamento transverso do úmero; D- tendão do músculo bíceps braquial; D- corpo do úmero 


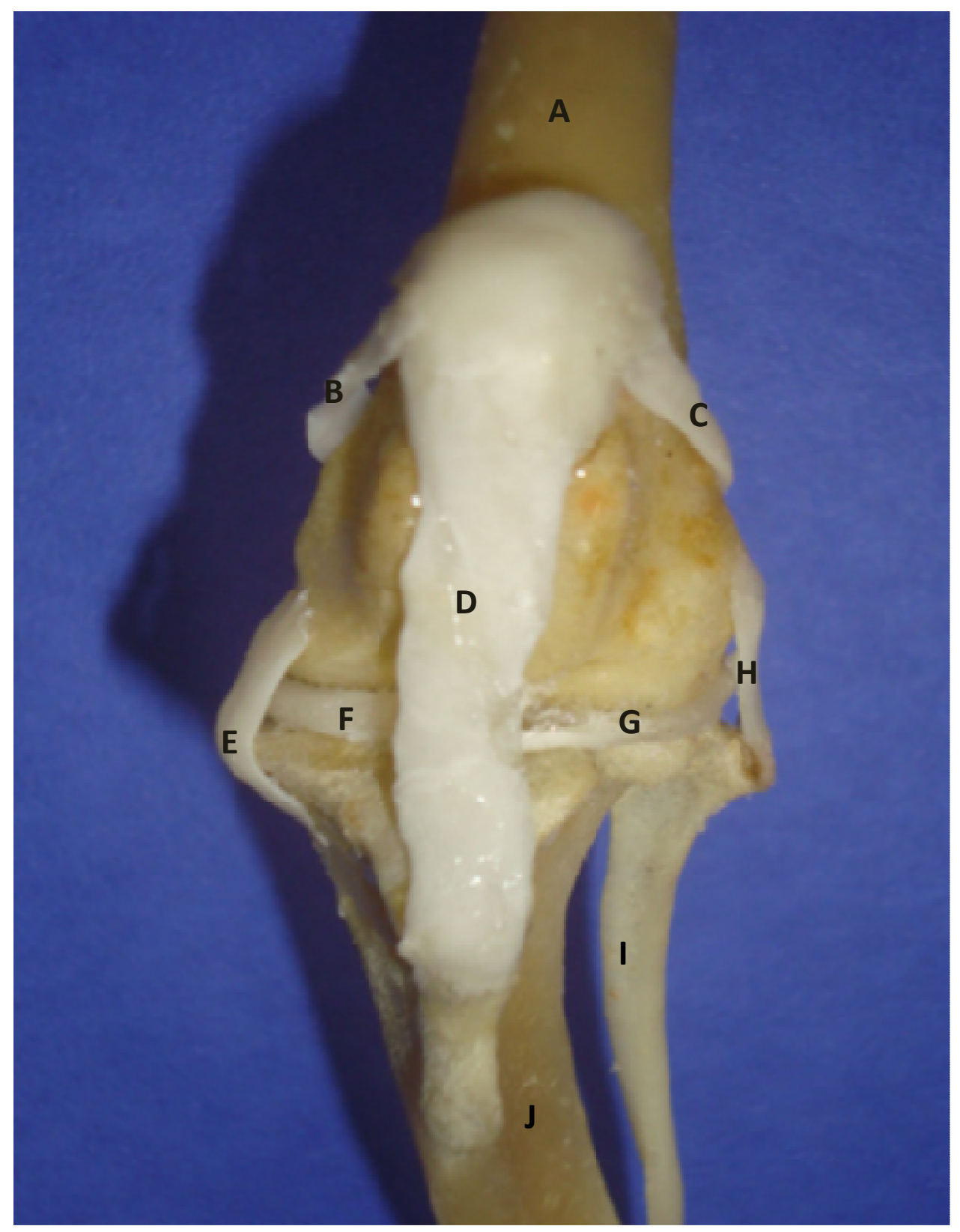

Figura 4: Articulação do joelho de cão: A- fêmur; Bligamento femoropatelar medial; C- ligamento femoropatelar lateral; D- ligamento patelar; E- ligamento colateral medial; F- Menisco medial; G- menisco lateral; $\mathrm{H}-$ ligamento colateral lateral; I-Fíbula; J- corpo da tíbia.

\section{Topografia vascular}

Uma análise da topografia vascular do sincrânio de um equino pode ser demonstrada com o uso de lã (vermelha) colada sobre a superfície óssea da 
face e do crânio de um equino (Figura 5), ilustrando desta forma todo o arranjo vascular arterial da referida região.

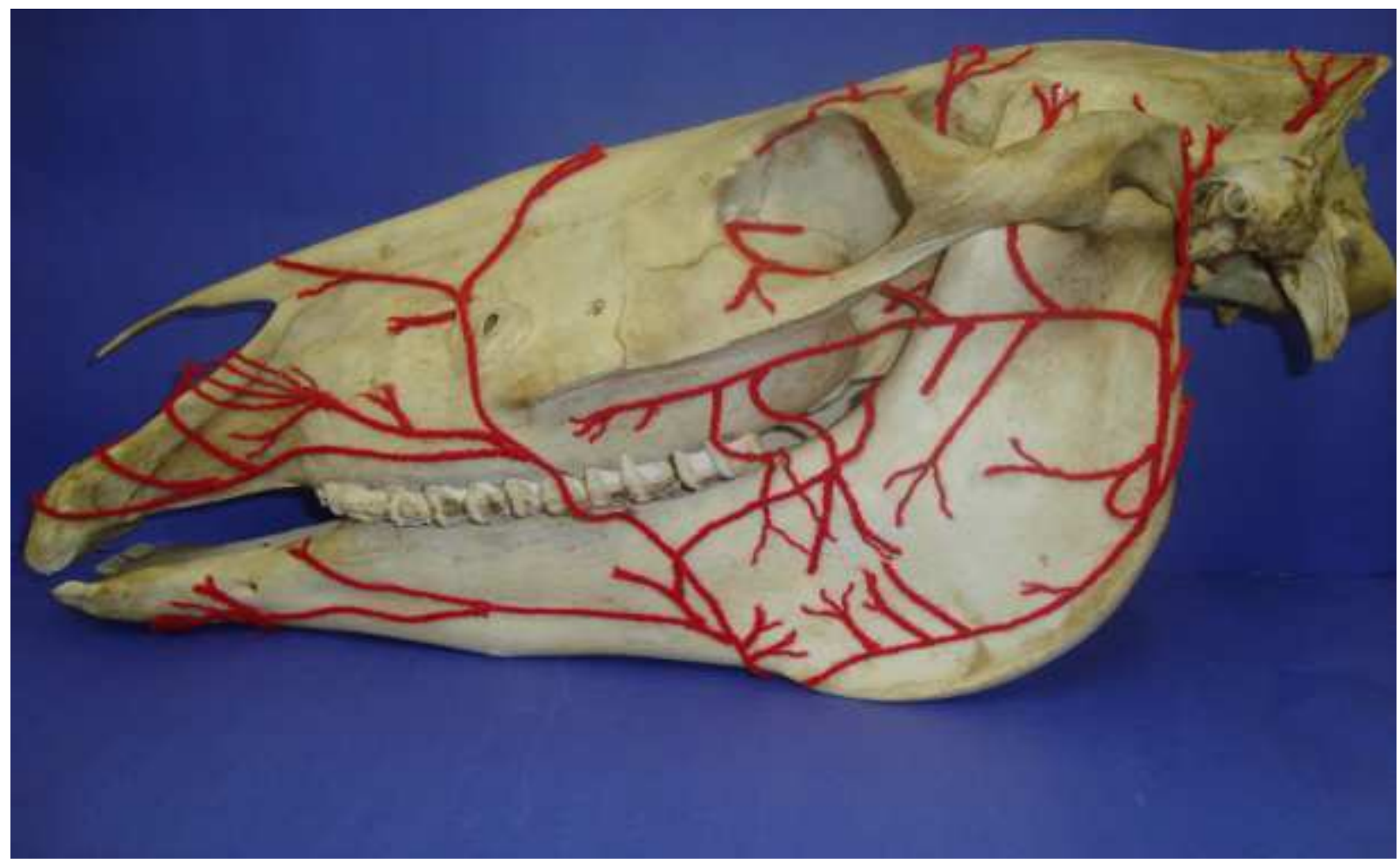

Figura 5: Vista esquerdo da topografia vascular arterial do sincrânio de eqüino.

Todas as peças apresentadas nesse artigo foram confeccionadas na Seção de Anatomia do Laboratório de Morfologia do Centro de Ciências e Tecnologias Agropecuárias da UENF e são amplamente utilizadas para fins didáticos nas aulas práticas e teóricas das disciplinas de Anatomia dos Animal Domésticos ministradas para os cursos de Medicina Veterinária e Zootecnia.

\section{Conclusão:}

A utilização destes recursos didáticos vem se mostrando um agente facilitador da aprendizagem, já que os alunos e professores tem a possibilidade de manusearem peças secas, sem o cheiro de formol e com uma vida útil bem maior. A implementação de novas metodologias de ensino, como as citadas neste trabalho, contribuíram de maneira positiva no processo ensino- 
SOUZA, A.L.M. et al. Utilização de métodos didáticos alternativos para o estudo da anatomia veterinária. PUBVET, Londrina, V. 6, N. 27, Ed. 214, Art. 1428, 2012.

aprendizagem da anatomia animal. Assim, é possível concluir que a utilização de métodos alternativos ao uso desnecessário de animais é ético, eficaz e economicamente viável.

\section{Referências Bibliográficas:}

MAGALHÃES, M.; ORTÊNCIO FILHO, H. Alternativas ao uso de animais como recurso didático. Arq. Ciênc. Vet. Zool. Unipar, Umuarama, v. 9, n. 2, p. 147-154, 2006.

MARTINSEN, S.; JUKES, N. Towards a humane veterinary education. Journal of Veterinary Medical Education, v. 32, n. 4, p. 454-460, 2005.

GAUTHIER, C.; GRIFFIN, G. Using animals in research, testing and teaching. Revue scientifi que et technique de I'Offi ce international des épizooties, v. 24, n. 2, p. 735-745, 2005.

SANTOS, C.R.O.; Amorim, M.M.A.; Melo, R.R.C.B.; Nadyne Lohayne Farias Rocha, N.L.F.C.; Barreto, M.L.M.; wanderley, S.R.S.; Silva, L.T.R.; Rolim, V.P.; Amorim, M.J.A.A.L. Uso de técnicas didático-práticas aplicadas por monitores de anatomia na aprendizagem dos planos de construnção corporal. x jornada de ensino, pesquisa e extensão - jepex 2010 - ufrpe: recife, 18 a 22 de outubro.

MARQUES, R. G. Rumo à regulamentação da utilização de animais no ensino e na pesquisa científica no Brasil. Acta Cirúrgica Brasileira, v. 20, n. 3, p. 262-267, maio/jun, 2005. 